\title{
Comparison of intraoperative MR imaging and 3D-navigated ultrasonography in the detection and resection control of lesions
}

\author{
Volker M. Tronnier, M.D., Ph.D., Matteo M. Bonsanto, M.D., \\ Andreas Staubert, DiPl. Phys., Michael Knauth, M.D., Ph.D., \\ Stefan Kunze, M.D., Ph.D. and Christian R. WirTz, M.D.
}

Departments of Neurological Surgery and Neuroradiology, University Hospital Heidelberg, Heidelberg College of Medicine, Heidelberg, Germany

\begin{abstract}
Object. The authors undertook a study to compare two intraoperative imaging modalities, low-field magnetic resonance (MR) imaging and a prototype of a three-dimensional (3D)-navigated ultrasonography in terms of imaging quality in lesion detection and intraoperative resection control.

Methods. Low-field MR imaging was used for intraoperative resection control and update of navigational data in 101 patients with supratentorial gliomas. Thirty-five patients with different lesions underwent surgery in which the prototype of a 3D-navigated ultrasonography system was used. A prospective comparative study of both intraoperative imaging modalities was initiated with the first seven cases presented here.

In 35 patients (70\%) in whom ultrasonography was performed, accurate tumor delineation was demonstrated prior to tumor resection. In the remaining $30 \%$ comparison of preoperative MR imaging data and ultrasonography data allowed sufficient anatomical localization to be achieved. Detection of metastases and high-grade gliomas and intraoperative delineation of tumor remnants were comparable between both imaging modalities. In one case of a low-grade glioma better visibility was achieved with ultrasonography. However, intraoperative findings after resection were still difficult to interpret with ultrasonography alone most likely due to the beginning of a learning curve.

Conclusions. Based on these preliminary results, intraoperative MR imaging remains superior to intraoperative ultrasonography in terms of resection control in glioma surgery. Nevertheless, the different features (different planes of slices, any-plane slicing, and creation of a 3D volume and matching of images) of this new ultrasonography system make this tool a very attractive alternative. The intended study of both imaging modalities will hopefully allow a comparison regarding sensitivity and specificity of intraoperative tumor remnant detection, as well as cost effectiveness.
\end{abstract}

\section{KEY WORDS • glioma • intraoperative magnetic resonance imaging • neuronavigation • ultrasonography}

Modern imaging technologies have a major impact on spinal and intracranial neurosurgery. Better visualization by the operating microscope has markedly enhanced intraoperative handling and patient safety. Endoscopic-assisted neurosurgery enables the surgeon to look "around the corner" into an internal meatus or around a brain vessel to ensure that a clip is correctly placed in aneurysm surgery. In the past decade, computer-assisted image-guided surgery (neuronavigation) has been developed to assist neurosurgeons in performing surgery more safely, efficaciously, and cost effectively. Neuronavigation allows the neurosurgeon to localize the lesion more accurately, to determine the lesion size, and to choose a safe surgical corridor by which to approach the lesion. Currently available systems include frame-based or frameless systems that are based on different localizing techniques as passive pointers, LED-integrated optical pointers, or electromag-

Abbreviations used in this paper: $\mathrm{CT}=$ computerized tomography; LED = light-emitting diode; $\mathrm{MR}=$ magnetic resonance; $3 \mathrm{D}=$ three-dimensional. netic systems. All these image-guided systems, however, require imaging data (CT and MR imaging as well as, more recently, integrated functional MR imaging, positron emission tomography, and magnetoencephalography) acquired preoperatively. None of these systems can provide surgeons with information about intraoperative dynamic changes such as brain shift due to loss of cerebrospinal fluid, tumor debulking, or brain deformation caused by patient positioning. Knowledge of these changes is crucial in determining the amount of brain tumor to be resected, which is one of the key factors of postoperative progression-free interval and survival. Recently, intraoperative MR imaging was introduced by our group and others ${ }^{2,7,15,16,20-27}$ to solve some of the aforementioned problems. Although excellent in discriminating a lesion from the surrounding brain by the presence of tissue contrast, MR imaging is a bulky device, interfering with many surgical instruments, including the operating microscope and many electronic systems in the operating room. Therefore, other groups prefer to use intraoperative ultrasonography $4,5,10,11-14,17,19,28$ as a real-time monitoring device, which is easier to handle and less costly. However, 


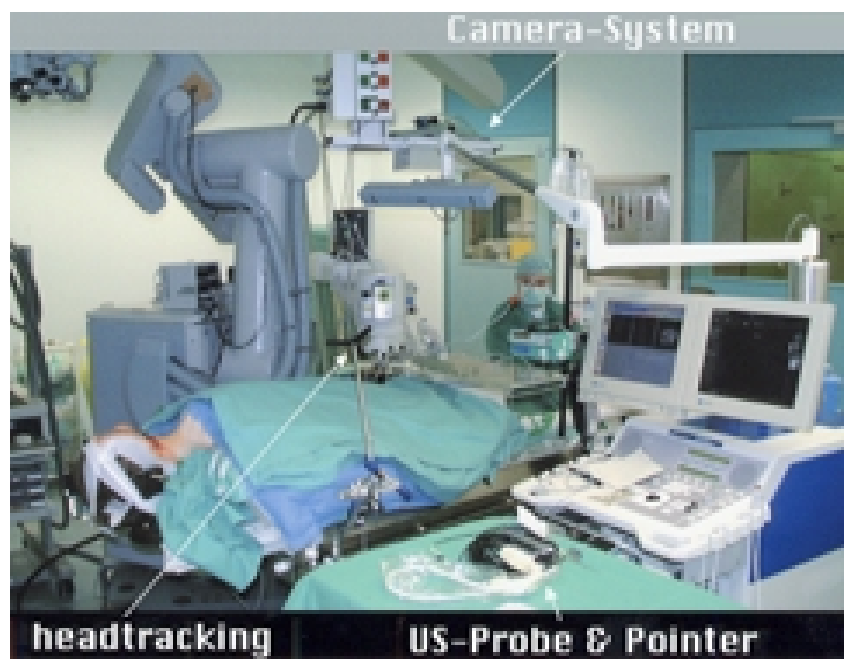

Fig. 1. Photograph showing the operative setup of the navigational system (left screen on the rack) and the ultrasonography system (right screen). A pointer and the ultrasonography probe can be tracked by the camera system.

many problems, such as correlating preoperative MR images with intraoperative ultrasonography images (that is, different data formats) and the evaluation of tumor remnants with high sensitivity and specificity, have not been solved yet. Based on our experience with 320 cases in which surgery was performed with the assistance of intraoperative MR imaging, we decided to undertake a prospective study to compare intraoperative MR imaging and high-end 3D ultrasonography. ${ }^{11}$ The first preliminary results are presented in this report.

\section{CLINICAL MATERIAL AND METHODS}

One hundred and one procedures were performed with the assistance of intraoperative MR imaging (0.2 tesla,

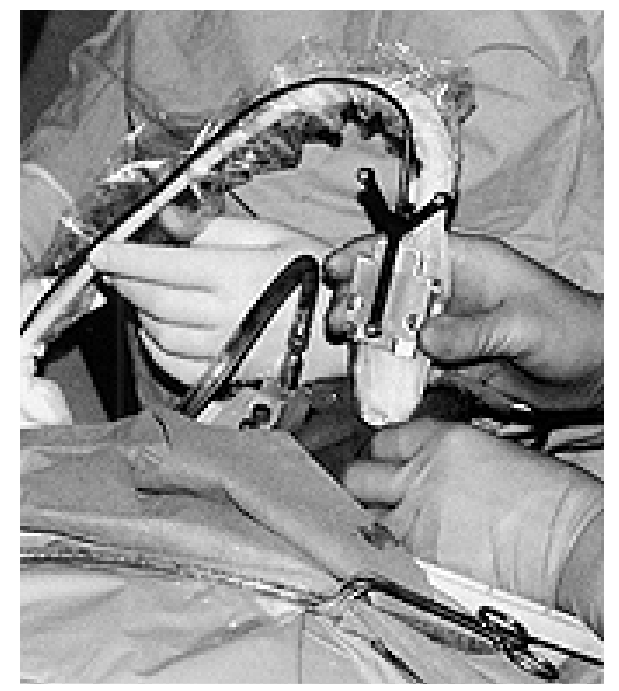

Fig. 2. The ultrasonography probe, with three active LEDs, allows localization of the probe and the imaging plane of the ultrasonography with correlation to preoperative images.
Magnetom Open; Siemens, Germany) and the update of navigational datasets for supratentorial gliomas. The postoperative course of these patients (minimum follow up 6 months, mean follow up $22.2 \pm 9.5$ months) was analyzed, as were intraoperative imaging quality, interpretational difficulties, and the extent of tumor removal. These data were presented in an earlier paper. ${ }^{26}$ Thirty-five patients underwent intraoperative 3D-navigated ultrasonography (UltraWand; Mison, Trondheim, Norway) equipped with a high-end ultrasonography scanner and a high-end G4 personal computer (G4 Power PC; Apple Computer, Cupertino, California, USA) with an active optical LEDbased positioning and digitizer system (Flashpoint 5000; Image-guided Technologies, Inc., Boulder, CO) (Fig. 1). This system is a prototype version of the final product SonoWand. ${ }^{11}$ Finally in 7 patients both imaging modalities were used simultaneously.

The integration of an ethernet link into the UltraWand allowed it to be used like other navigational devices, importing imaging data from the neuroradiological suite. A direct link beween the ultrasonography unit and the navigation system allows for the rapid transfer of the imaging information without loss of quality. A 4 to $8-\mathrm{MHz}$ phased array probe allowed overview images and the creation of freehand 3D images. The ultrasonography probe and the pointer are linked to active LEDs and can be tracked by the camera system (Fig. 2).

The 3D images are created by tilting the ultrasonography probe over $90^{\circ}$ within 15 seconds. A pyramid-shaped volume of the region of interest is acquired and can be compared with pre- or intraoperative MR images. With two-dimensional real-time ultrasonography data slices in any direction are created and can be compared with the corresponding CT or MR images (Figs. 3-5). Additionally

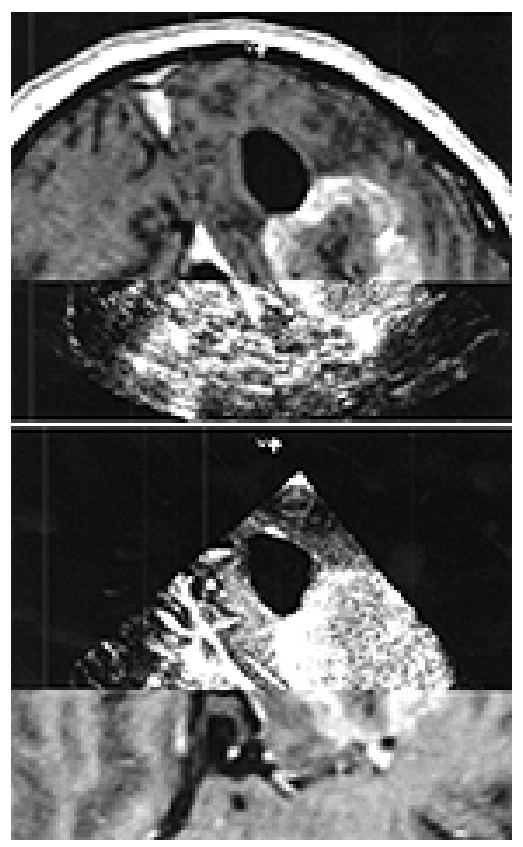

Fig. 3. Upper and Lower: "Any-plane slicing." An overlay of two 3D volumes obtained from MR and ultrasound images with arbitrary cross sections not restricted to a certain plane. 


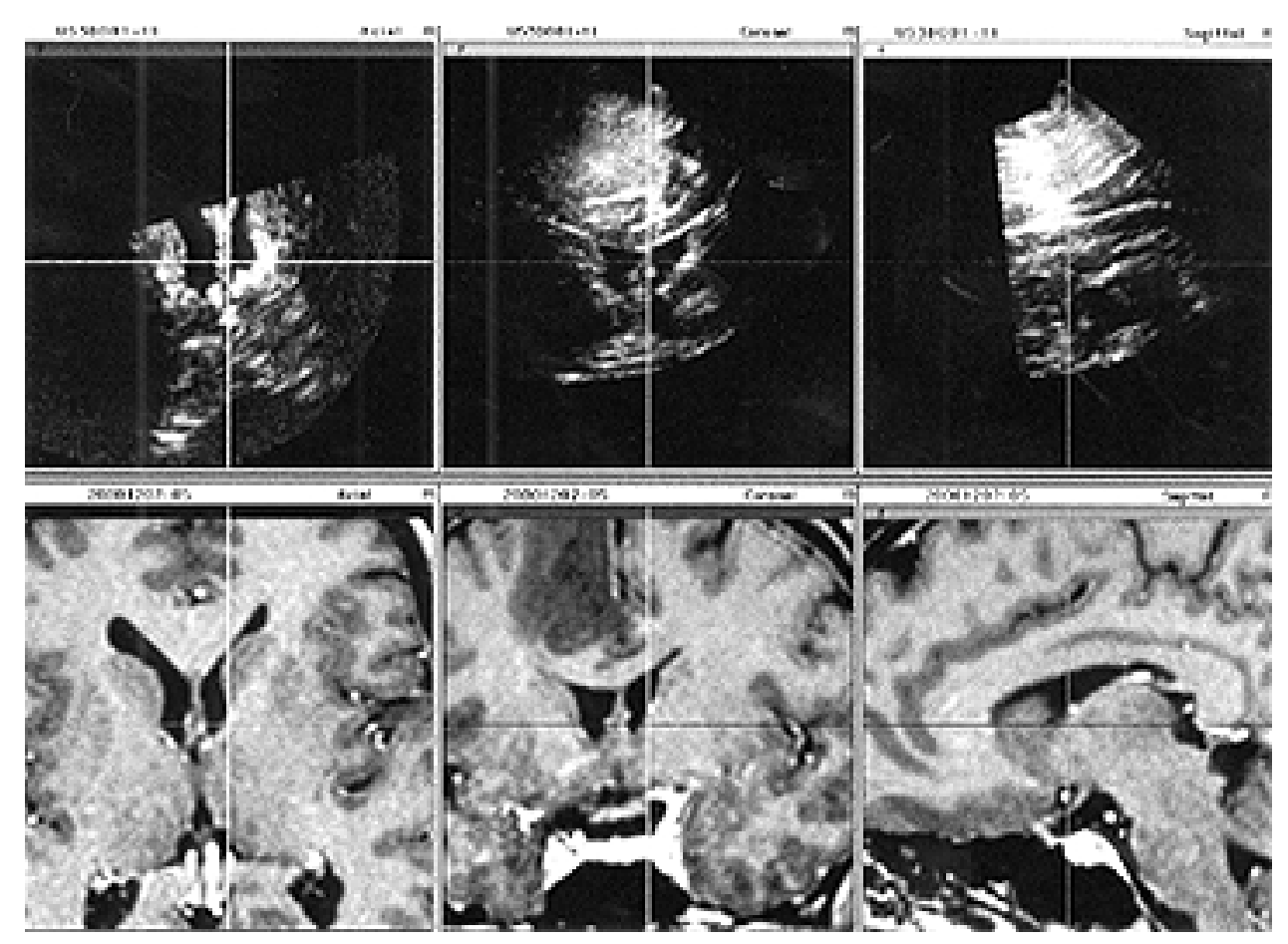

Fig. 4. "Orthogonal slicing" in three different planes. The images demonstrate a left frontal precentral low-grade glioma with exact anatomical correspondence of the tumor borders in the coronal section; however, there is a suspicious hyperechogenic area in the sagittal plane of the ultrasound in contrast to the MR image.

a pointer can be used, as in other navigational systems, which is displayed as a dashed line on the corresponding images and demonstrates the actual position of the instrument within the MR image and the 3D ultrasonography datasets. Blood vessels are easily detected using the flow mode of the ultrasonography unit.

\section{RESULTS}

\section{Tumor Detection Prior to Resection}

There were no intraoperative complications related to either imaging procedure. Imaging quality was good or fair in $85.5 \%$ (101) of the cases in which MR imaging was used and in $70 \%$ (35) of the cases in which ultrasonography was performed. Good imaging quality was defined as exact tumor delineation, as judged by three different readers (two surgeons and one radiologist). Fair imaging quality was considered to have been obtained in cases in which the tumor margins could not be delineated with certainty. In $30 \%$ of the cases the UltraWand was used as a pure neuronavigational tool. In another $30 \%$ of the cases it was difficult to delineate anatomical landmarks by ultrasonography alone. The combination and matching with preoperative MR imaging data allowed sufficient anatomical localization. The optical tracking system showed a high reliability. The main advantage, however, is that the device can be used as a navigational system, as a pure ultrasonography imager, or in combination.

\section{Intraoperative Resection Control}

The creation of a $3 \mathrm{D}$ volume, which is used to match intraoperative data with preoperative MR imaging data, demonstrated sufficiently intraoperative brain shift. Therefore, intraoperative resection control in glioma surgery or other procedures is feasible. Good imaging quality was considered to have been obtained in cases in which there was certain delineation of tumor remnants or tumorfree resection area. The few combined studies revealed visualization of low-grade tumors was increased on ultrasound images; a case with a pilocytic astrocytoma of the posterior fossa was not well detected by ultrasonography. In metastases (Fig. 3) and high-grade gliomas (Fig. 5) the primary visualization of ultrasonography is comparable with intraoperative MR imaging. However, the interpretation of ultrasound images during the resection requires improvement. In three of our cases in which combined imaging was performed, tumor remnants were less visible on intraoperative ultrasound images compared with intraoperative MR images. Further, in one case the resection border was considered inconspicuous on intraoperative ultrasonography, whereas a biopsy sample-proven tumor remnant could be detected by intraoperative MR imaging. This may be in part due to the fact of the learning curve: neurosurgeons are more inclined to interpret pre- and intraoperative CT or MR images than ultrasound images.

\section{DISCUSSION}

Ultrasonography as a diagnostic tool is widely used in neurosurgery for the detection of deep-seated lesions. However, its use in intraoperative resection was disregarded because of its low-quality imaging capabilities in 


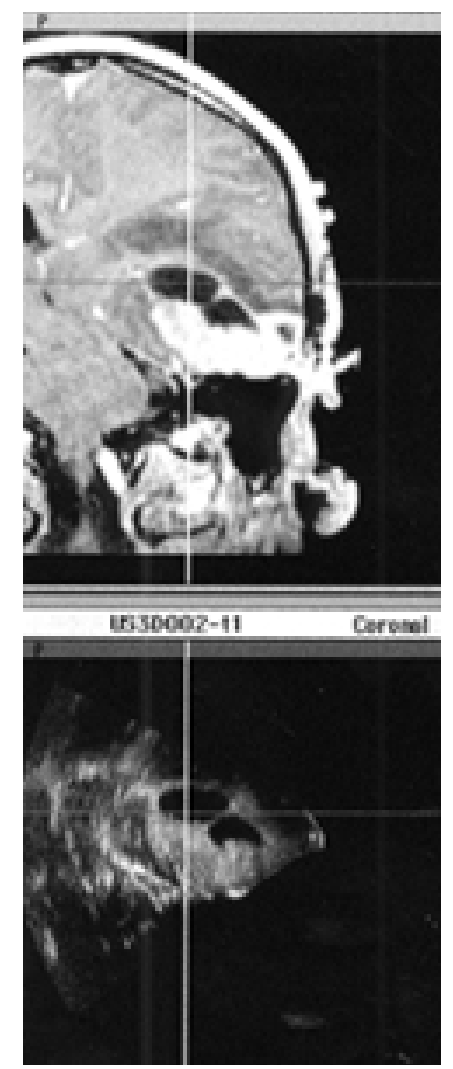

Fig. 5. Images obtained in a 65-year-old man with right temporal glioblastoma. Good ultrasound/MR image correlation between necrotic and solid tumor areas is apparent.

the past two decades. Computerized tomography and MR imaging-guided computer-assisted surgery, or neuronavigation, has evolved since the mid-1980s. However, several researchers have attempted to combine intraoperative neuronavigation and ultrasonography to obtain real-time control during surgery. At present high-end ultrasonography systems provide the surgeons with excellent imaging quality, ${ }^{11-14,19,28}$ although neurosurgeons are usually still more prone to read and interpret CT or MR images. The extent of tumor resection has been recognized as one of the factors determining a patient's survival, and this is true in cases of high-grade gliomas ${ }^{1,9,18}$ but especially in those of low-grade gliomas and pediatric tumors. ${ }^{3,6,8}$ Although navigational systems enhance the completeness of tumor resection to some extent, ${ }^{24}$ the ability to determine intraoperative resection control was a logical progression for neurosurgeons. Currently several competitive concepts exist. Although CT scanning in general is not recommended because of the use of ionizing radiation, low tissue contrast, and problems in differentiating blood in the resection cavity or resection margin from any residual tumor, introperative ultrasonography might be an attractive alternative to intraoperative MR imaging. Restriction of space in the operating room and lower costs as compared with MR imaging are additional advantages. Hammoud, et al., ${ }^{12}$ conducted a prospective study of 34 patients harboring metastatic tumors and 36 patients harboring gliomas, comparing the use of intraoperative ultrasonogra- phy and postoperative MR imaging. They found that ultrasonography provided a good delineation of the tumor border in $83 \%$ of the primary gliomas and in $60 \%$ of the recurrent tumors. However, in patients who had previously undergone irradiation, tumor localization was difficult in $38 \%$, and determination of the tumor border in $85 \%$. Metastatic lesions were easily localized and their margins detected. The extent of resection was well defined in all of their patients (in five of 18 patients, however, only subtotal tumor resection was achieved), in $80 \%$ of the recurrent gliomas and in none of the patients who had undergone irradiation. Still unsolved is the question of which imaging modality demonstrates higher sensitivity and specificity in the detection of tumor remnants. Therefore, comparative studies are mandatory. Mäurer, et al., ${ }^{19}$ performed a comparative study of early postoperative MR imaging, CT scanning, and transcranial sonography findings. They conducted early postoperative CT scanning in 73 patients with high-grade gliomas in which residual tumor at the resection site was demonstrated by contrast enhancement in 41 patients $(56.2 \%)$; MR imaging was performed in only 35 patients, with early postoperative nonlinear contrast enhancement observed in 24 patients $(68.6 \%)$. In contrast, transcranial sonography revealed a nonlinear hyperechogenic area in $95.9 \%$ of their cases, with a volume that exceeded on average the CT-detected volume by $4.4 \mathrm{~cm}^{2}$ and MR imaging-detected volume by $3.3 \mathrm{~cm}^{2}$. The survival rate of patients in whom no enhancement was demonstrated on postoperative MR imaging correlated with significantly longer survival. This finding is in accordance with those of our earlier studies. ${ }^{1,26}$ Additionally patients in whom a smaller hyperechogenic area was observed $(<$ $4 \mathrm{~cm}^{2}$ ) on postoperative transcranial sonography had a significantly longer survival rate than those with larger tumor remnants. They concluded that the sensitivity of transcranial sonography exceeds that of CT and matches that of MR imaging but that the disadvantage is a lower specificity. Therefore, a prospective comparative study of both imaging modalities performed intraoperatively as well as confirmation of the findings by histological examination are mandatory.

\section{CONCLUSIONS}

Intraoperative ultrasonography and MR imaging may provide the surgeon with valuable information regarding resection control of intraaxial brain neoplasms. After several feasibility studies and technical reports of intraoperative MR imaging and intraoperative ultrasonography, more critical analyses of long-term outcome, side-effects, and cost-benefit ratios are needed. A comparative study will demonstrate the advantages and limitations of both imaging modalities.

\section{References}

1. Albert FK, Forsting M, Sartor K, et al: Early postoperative magnetic resonance imaging after resection of malignant glioma: objective evaluation of residual tumour and its influence on regrowth and prognosis. Neurosurgery 34:45-61, 1994

2. Albert FK, Tronnier VM, Wirtz CR, et al: Intraoperative diagnostic and interventional MR in neurosurgery: first experiences with an "open MR" system, in Hellwig D, Bauer BL (eds): 
Minimally Invasive Techniques for Neurosurgery: Current Status and Future Perspectives. Heidelberg: Springer-Verlag, 1998, pp 229-235

3. Albright AL, Wisoff JH, Zeltzer PM, et al: Effects of medulloblastoma resections on outcome in children: a report from the Children's Cancer Group. Neurosurgery 38:265-271, 1996

4. Auer LM, van Velthoven V: Intraoperative ultrasound (US) imaging. Comparison of pathomorphological findings in US and CT. Acta Neurochir 104:84-95, 1990

5. Becker G, Hofmann E, Woydt M, et al: Postoperative neuroimaging of high grade gliomas: comparison of transcranial sonography, magnetic resonance imaging, and computed tomography. Neurosurgery 44:469-478, 1999

6. Berger MS, Deliganis AV, Dobbins J, et al: The effect of extent of resection on recurrence in patients with low grade cerebral hemisphere gliomas. Cancer 74:1784-1791, 1994

7. Black PM, Moriarty T, Alexander E III, et al: Development and implementation of intraoperative magnetic resonance imaging and its neurosurgical applications. Neurosurgery 41:831-845, 1997

8. Bourne JP, Geyer R, Berger M, et al: The prognostic significance of postoperative residual contrast enhancement on CT scan in pediatric patients with medulloblastoma. J Neurooncol 14:263-270, 1992

9. Campbell JW, Pollack IF, Martinez AJ, et al: High-grade astrocytomas in children: radiologically complete resection is associated with an excellent long-term prognosis. Neurosurgery 38:258-264, 1996

10. Chandler WF, Knake JE, McGillicuddy JE, et al: Intraoperative use of real-time ultrasonography in neurosurgery. J Neurosurg 57:157-163, 1982

11. Gronningsaeter A, Kleven A, Ommedal S, et al: SonoWand, an ultrasound-based neuronavigation system. Neurosurgery 47: 1373-1380, 2000

12. Hammoud MA, Ligon BL, elSouki R, et al: Use of intraoperative ultrasound for localizing tumors and determining the amount of resection: a comparative study with magnetic resonance imaging. J Neurosurg 84:737-741, 1996

13. Hata N, Dohi T, Iseki H, et al: Development of a frameless and armless stereotactic neuronavigation system with ultrasonographic registration. Neurosurgery 41:608-614, 1997

14. Jödicke A, Deinsberger W, Erbe H, et al: Intraoperative threedimensional ultrasonography: an approach to register brain shift using multidimensional image processing. Minim Invasive Neurosurg 41:13-19, 1998

15. Knauth M, Wirtz CR, Tronnier VM, et al: [Intraoperative magnetic resonance tomography for control of extent of neurosurgical operations.] Radiologe 38:218-224, 1998 (Ger)

16. Knauth M, Wirtz CR, Tronnier VM, et al: Intraoperative MR imaging increases the extent of tumor resection in patients with high-grade gliomas. AJNR 20:1642-1646, 1999
17. Koivukangas J, Louhisalmi Y, Alakuijala J, et al: Ultrasoundcontrolled neuronavigator-guided brain surgery. J Neurosurg 79:36-42, 1993

18. Kowalczuk A, Macdonald RL, Amidei C, et al: Quantitative imaging study of extent of surgical resection and prognosis of malignant astrocytomas. Neurosurgery 41:1028-1038, 1997

19. Mäurer M, Becker G, Wagner R, et al: Early postoperative transcranial sonography (TCS), CT, and MRI after resection of high grade glioma: evaluation of residual tumour and its influence on prognosis. Acta neurochir 142:1089-1097, 2000

20. Steinmeier R, Fahlbusch R, Ganslandt O, et al: Intraoperative magnetic resonance imaging with the magnetom open scanner: concepts, neurosurgical indications, and procedures: a preliminary report. Neurosurgery 43:739-747, 1998

21. Sutherland G, Kaibara T, Louw D, et al: A mobile high-field magnetic resonance system for neurosurgery. J Neurosurg 91: 804-813, 1999

22. Tronnier VM, Wirtz CR, Knauth M, et al: Intraoperative diagnostic and interventional magnetic resonance imaging in neurosurgery. Neurosurgery 40:891-902, 1997

23. Tronnier V, Wirtz CR, Staubert A, et al: Recent development and experiences of intraoperative MRI, in Tamaki N, Ehara K (eds): Brain Tumors of Maldevelopmental Origin. Osaka: Medicus Shuppan, 1999, pp 211-222

24. Wirtz CR, Albert FK, Schwaderer M, et al: The benefit of neuronavigation for neurosurgery analyzed by its impact on glioblastoma surgery. Neurol Res 22:354-360, 2000

25. Wirtz CR, Bonsanto MM, Knauth M, et al: Intraoperative magnetic resonance imaging to update interactive navigation in neurosurgery: method and preliminary experience. Comput Aided Surg 2:172-179, 1997

26. Wirtz CR, Knauth M, Staubert A, et al: Clinical evaluation and follow-up results for intraoperative magnetic resonance imaging in neurosurgery. Neurosurgery 46:1112-1122, 2000

27. Wirtz CR, Tronnier VM, Bonsanto MM, et al: Image-guided neurosurgery with intraoperative MRI: update of frameless stereotaxy and radicality control. Stereotact Funct Neurosurg 68:39-43, 1997

28. Woydt M, Krone A, Becker G, et al: Correlation of intra-operative ultrasound with histopathologic findings after tumour resection in supratentorial gliomas. A method to improve gross total tumour resection. Acta Neurochir 138:1391-1398, 1996

Manuscript received December 15, 2000.

Accepted in final form January 26, 2001.

Address reprint requests to: Volker M. Tronnier, M.D., Ph.D., Department of Neurosurgery, University Hospital Heidelberg, Im Neuenheimer Feld 400, D-69120 Heidelberg, Germany. email: volker_tronnier@med.uni-heidelberg.de. 